\title{
Ciencia, desarrollo humano y calidad de vida
}

\author{
Rocío Calderón García*
}

Recibido: 7 de septiembre de 2009 Revisado: 23 de septiembre de 2009 Aprobado: 5 de octubre de 2009

\section{RESUMEN}

En la sociedad contemporánea, la ciencia y la tecnología se han convertido en el centro alrededor del cual se entretejen las relaciones sociales y la vida social. La sociedad del conocimiento, como se le ha denominado al modelo de la sociedad a la que aspiraríamos, precisamente por el desarrollo que ha tenido el conocimiento tecnológico y científico en estos momentos, se convierte en el ámbito natural para que el tema de la tecnociencia forme parte de los procesos formativos y constitutivos de las nuevas identidades sociales. El hecho de entender como la población incorpora los conceptos de ciencia y tecnología en sus agendas de discusión en la toma de decisiones sobre su vida y en sus actividades cotidianas, se convierte hoy en día en una explicación necesaria para entender los procesos de formación y expresión del ser humano contemporáneo.

Palabras clave: ciencia, calidad de vida y desarrollo humano.

* Doctora en Cooperación y Bienestar Social por la Universidad de Oviedo, España, Profesora e Investigadora del Departamento de Estudios Internacionales de la Universidad de Guadalajara, Miembro de la Academia J alisciense de Ciencia y del Sistema Nacional de Investigadores. Correo electrónico: hallazgos@ usantotomas.edu.co. 


\title{
Science, human development and life quality
}

\author{
Rocío Calderón García
}

\begin{abstract}
Science and technology in contemporary society have become the center around which social relations and social life are interlaced. A society of knowledge, as we have named the model society which we aspire to, precisely due to the development that technical and scientific knowledge has had in recent times, becomes a natural forum where the subject of techno-science is part of the formative processes and constitute part of the new social identities. The understanding of how the population incorporates the concepts of science and technology into its agendas of discussion, in making decisions regarding life and daily activities becomes today a necessary explanation in order to understand the processes of formation and expression of the contemporary human being.
\end{abstract} Aprobado: 5 de octubre de 2009

\section{Key Words}

Science, Quality of life, Human development. 


\section{INTRODUCCIÓN}

A nivel mundial, el actual escenario se caracteriza por ser altamente turbulento; en éste, los cambios son continuos, todo está en permanente transformación. Exige de los países mayores niveles de competitividad. Ahora bien, un país la alcanzará si al mismo tiempo logra consolidar su estabilidad macroeconómica, su institucionalidad y desarrollar sobre todo la ciencia y tecnología.

Hoy más que nunca, la ciencia y la tecnología son herramientas indispensables en la construcción de sociedades modernas e incluyentes. El fortalecimiento de la investigación científica y la innovación tecnológica son tareas imprescindibles para apoyar el desarrollo del país y para competir en un entorno cada vez más dominado por el conocimiento y la información. Siendo el conocimiento científico y técnico, su producción y su aplicación a todos los ámbitos de la vida de nuestras sociedades, una de las fuerzas motrices de los procesos de crecimiento económico y de mejora del bienestar social.

Dentro del marco normativo que regula la actividad en ciencia y tecnología en México tenemos, en primer término, la Constitución Política de los Estados Unidos Mexicanos, la cual en su Artículo 3, Fracción V, dispone que el Estado, además de impartir la educación preescolar, primaria y secundaria, promoverá y atenderá todos los tipos y modalidades educativos -incluyendo la educación superior- necesarios para el desarrollo de la Nación; asimismo, apoyará la investigación científica y tecnológica, y alentará el fortalecimiento y difusión de nuestra cultura.
Derivado de lo anterior tenemos que el 29 de diciembre de 1970 se creó el Consejo Nacional de Ciencia y Tecnología por disposición del H. Congreso de la Unión como un organismo público descentralizado de la Administración Pública Federal, integrante del Sector Educativo, con personalidad jurídica y patrimonio propio.

Este organismo es el responsable de elaborar las políticas de ciencia y tecnología en México. Desde su creación hasta 1999, se presentaron dos reformas y una ley para coordinar y promover el desarrollo científico y tecnológico. El 5 de junio del 2002 se promulgó una nueva Ley de Ciencia y Tecnología; en ésta, publicada en el Diario Oficial de la Federación, el 5 de junio del 2002, en su capítulo III (Principios orientadores del apoyo a la actividad científica y tecnológica), en su Artículo XII establece que "Se promoverá la divulgación de la ciencia y la tecnología con el propósito de ampliar y fortalecer la cultura científica y tecnológica en la sociedad". También se indica que el Gobierno del Estado de Jalisco y el Consejo Estatal de Ciencia y Tecnología (CONACYT), con base en los dispuesto en la Ley de Ciencia y Tecnología, han constituido un fideicomiso con recursos concurrentes denominado "Fondo Mixto CONACYT-Gobierno del Estado de Jalisco", siendo uno de los propósitos atender problemas, necesidades y oportunidades del Estado.

En este contexto en el Plan Nacional de Desarrollo 2007-2012 se destaca que:

[...] la investigación científica, así como el desarrollo y la innovación tecnológica son precursores esenciales de la competitividad y el crecimiento económico, la presente administración se ha propuesto darles fuerte impulso, 
promoviendo vínculos más profundos y estrechos entre el sector productivo y los centros educativos y de investigación, además de favorecer el desarrollo de mecanismos de financiamiento adicionales a la asignación directa de recursos fiscales, así como asegurar que los recursos públicos se canalicen a áreas prioritarias para el país.

En el Plan Estatal de Desarrollo del Estado de Jalisco con visión a 2030, se establece que la ciencia y la tecnología y el desarrollo de Jalisco estarán en estrecha vinculación; así el Programa Sectorial 2007-2013 señala: “la ciencia, la tecnología y la innovación son elementos básicos en el desarrollo económico y social de un estado y sus regiones". Además, enfatiza que: "el aspecto social, la ciencia, la tecnología y la innovación juegan un papel fundamental en la movilidad social, los niveles educativos y la generación de oportunidades que caracterizan a las nuevas sociedades basadas en el conocimiento". Asimismo, relaciona el presupuesto de ciencia y tecnología del año 2007 como porcentaje del PIB estatal, con respecto a la meta planteada para el año 2013 cuantificada en $0,30 \%$; en consecuencia, existe un incremento del $0,59 \%$.

Para el año 2008 el presupuesto asignado es de 236 millones de pesos, con un porcentaje de incremento de $168 \%$ con respecto al año 2007; esto representa 1,99\% del presupuesto federal asignado para ciencia y tecnología que asciende de 11 millones a 876 mil pesos.

Con respecto al tamaño de la economía en el estado de Jalisco, medido por el valor del Producto Interno Bruto (PIB), las erogaciones realizadas en ciencia y tecnología, en el año 2006 representaron un 0,0120\%, mientras en el año 2007, las aportaciones al presupuesto en ciencia y tecnología admi- nistradas por el COECYTJAL significan un 0,0137\% del valor del PIB en ese año, lo cual muestra un avance en año 2007 del 14\%, en relación a la proporción observada en 2006.

En 2007 se incrementó en un 62\% el número de las instituciones académicas y empresas proponentes de proyectos en ciencia, tecnología e innovación, con respecto al año 2006 (de 64 a 104). Asimismo, se ha logrado la formación de redes institucionales de acuerdo con las necesidades del sector productivo y social. Tal es el caso del grupo de homólogos del sector de tecnologías de la información, microelectrónica y multimedia.

Es así como en el ámbito económico, el conocimiento es fuente crucial de valor añadido en la producción de bienes y servicios. Fenómenos tan variados como la extraordinaria productividad de los vegetales transgénicos, la efectividad de los medicamentos, o el rápido envejecimiento de los teléfonos móviles, el software, junto con el asesoramiento especializado muestran ese papel central del conocimiento en el mundo productivo. Pero también en la política pública éste juega un rol decisivo con la creciente institucionalización del consejo científico en los ámbitos más diversos, ya sea salud pública, obras públicas, agricultura, educación, cultura o deporte.

En este marco, dos de los grandes desafíos de la sociedad del conocimiento son, por un lado, la apropiación de ese conocimiento por el sistema productivo y, por otro, su apropiación por la sociedad civil.

La historia de la humanidad puede ser vista como una permanente búsqueda del conocimiento para comprender la realidad, y así modificarla en beneficio de los individuos, 
grupos o poblaciones. El uso manual de un objeto, el fuego, la rueda o los fundamentos de la agricultura rudimentaria fueron avances extraordinarios en esa búsqueda, antes de que se establecieran reglas y procedimientos generales para la observación y el análisis de datos e información que caracterizan el conocimiento científico.

Este tipo de conocimiento implica un progreso o avance en un campo disciplinar, ello impacta en la vida del ser humano, puede innovar en la solución de necesidades y hacer más llevadera la vida en sociedad, pero lo ideal será que este conocimiento lleve a la armonía tanto del sujeto como de la sociedad. Que los conocimientos no provoquen la insatisfacción del individuo; la manera en que se equilibren avances científicos, desarrollo y calidad de vida son los retos que un gobierno se puede plantear para el diseño y evaluación de políticas públicas que vayan orientadas hacia este objetivo, el bienestar de los individuos y su mejor inserción social.

\section{El papel de la ciencia}

La investigación precede a la ciencia. Sin embargo, la creación del método (una forma de organizar) es una plataforma desde la cual la administración del conocimiento ha dado saltos cada vez mayores. El conocimiento ya no depende exclusivamente de la "genialidad" o perseverancia individual y del "descubrimiento".

La construcción de una memoria sienta la base para la acumulación, la cual, a su vez, exige más y más la articulación de esfuerzos y multiplica la capacidad de creación. En este sentido Munch L y Angeles E (2005) indican que la espiral virtuosa se expande continuamente, alimentada también por demandas y apoyos sociales en expansión.
Aun en los periodos en que los valores e instituciones sociales prevalentes coartaron la libertad de pensar y de crear -como en la Edad Media y durante el orden feudal-, la fuerza modificadora de la búsqueda del conocimiento y de su utilización estuvo presente, alimentando las transformaciones del modo de producción y la estructuración de las ideas que finalmente rompieron las amarras del viejo orden.

Algunas conclusiones claras resultan de esa mirada a la historia:

- La producción y la utilización del conocimiento son funciones sociales: responden a demandas sociales expresadas de diferentes maneras que las legitiman y las hacen posibles.

- Es posible y necesario organizar los esfuerzos para la producción y la utilización del conocimiento. La organización legitimada socialmente potencia la capacidad de creación y aunque discipline, en ciertos parámetros, la libertad individual de investigación, al final incrementa su eficacia y la estimula.

- El desarrollo de la ciencia y de la técnica es promovido dentro de cada contexto social y económico.

- Hoy vivimos la plenitud de ese proceso. La ciencia y la técnica son los motores principales de la producción. La competitividad y la productividad son otros nombres de la innovación.

\section{EL PROGRESO Y LA INNOVACIÓN}

Cada veinte meses una tercera parte de la tecnología en uso se vuelve obsoleta y debe ser sustituida por otra que, muchas veces, ni siquiera se había previsto anteriormente. Los instrumentos de investigación son cada 
vez más potentes y los investigadores están mejor preparados y son más numerosos; la actividad científica es más compleja y más productiva, y cuenta con más recursos.

Esta situación abre un mundo de promesas para la humanidad, pero, al mismo tiempo, plantea dudas y amenazas. Entre ellas las que se refieren a la propiedad y al acceso al conocimiento, a la tecnología y a los bienes y servicios producidos con ellos. En un mundo globalizado por intereses económicos y estructurado sobre los principios del mercado, la competencia impone más rigor en la aceptación de la propiedad intelectual que, de hecho, estará bien protegida en los acuerdos multilaterales y en la legislación nacional resultante de éstos.

Las sociedades "marginales" o pobres, con una participación casi nula en el proceso de creación científica moderna, tendrán un acceso más difícil y más costoso a los resultados de esa creación. Más grave todavía, sus problemas específicos estarán relegados y olvidados y quedarán sin solución.

La dependencia es definitiva y las posibilidades de desarrollo son menores y condicionadas. Ese dilema está presente también en el área de la salud. Es en ese contexto global de promesas milagrosas y de dolorosa amenaza de dependencia permanente y marginación que se plantea la situación de la actividad científica en América Latina y el Caribe, especialmente, en relación con la salud. Las enseñanzas de la historia del conocimiento son cruciales para encontrar posibles soluciones.

El mundo moderno exige respuestas inmediatas y continuas que logren que el ser humano se enfrente consigo mismo y con sus valores. Se necesita pensar en el ser humano como persona que siente, sufre, que goza y. sobre todo, como un ser libre trascendental, espiritual, digno de respeto y reconocimiento.

De acuerdo con los estudios realizados por Méndez C. (2005), se debería reconocer que ninguna forma específica de conocimiento puede hacer justicia a toda la gama y complejidad de la experiencia humana, pues si no se admite esto, continuará el debate infructuoso y estéril entre los humanistas y los científicos. La investigación científica es tan humanista como la filosofía y la literatura. El ser humano ha sido siempre y al mismo tiempo hombre de letras, hombre filosófico. Por ello el reconocimiento de este hecho, es el tipo de conocimiento que se necesita en una edad o época como la actual, cuando el ser humano parece vivir un vacío existencial y en la cual el uso de la ciencia parece no tener conciencia.

Una ciencia con conciencia, esto es, con ideologías e instrumentos que se utilicen de manera correcta. No hay que olvidar como menciona Umberto Eco (2000) que así como la ciencia ha generado progreso, también ha generado destrucción y aniquilamiento. Según Jaki, el uso inadecuado de la ciencia puede convertir a ésta en una empresa "sin alma", en una aniquiladora del alma, si se le lleva a la categoría de filosofía fundamental, como parece suceder hoy, cuando el ser humano está al servicio de la ciencia y no la ciencia a su servicio.

Por eso la imperiosa necesidad de una ética en la ciencia, de manera que ésta, vaya orientada a formar mejores personas, más humanas y respetuosas de sí mismas, de los demás y del medio donde viven. 
Popper consideraba que la Ciencia, más que una teoría del conocimiento, se debe concebir como una actitud de respeto a la vida humana y a la moral, y propender al bienestar, procurando el mejoramiento de las condiciones de vida de toda la sociedad en pro de un mundo mejor.

\section{Ciencia y CAlidad de Vida}

De acuerdo con lo anterior, en un intento de aproximación hacia un concepto de calidad de vida, que se integre de las distintas dimensiones, como la dimensión física, social o mental, incluidas en las definiciones de "salud", "estado de salud" y "nivel de vida", todo ello desde el punto de vista de Augsto Bernal (2006) fundamentalmente subjetivo, por cuanto se tiene en cuenta esencialmente la percepción que el individuo experimenta directamente.

En este mismo sentido René Dubos nos señala que la "salud es aquel estado de la vida basado en un relativo equilibrio psíquico y unas funciones orgánicas intactas que permite a la persona llevar a cabo objetivos propios o ajenos por sus propias acciones".

Generalmente, nos indica Zapata O. (2005) que los componentes utilizados habitualmente en las herramientas para la evaluación de la calidad de vida se corresponden con las dimensiones definidas por la Organización Mundial de la Salud: Bienestar físico, bienestar emocional y bienestar social, considerando fundamentalmente las variables relacionadas con las condiciones de salud/enfermedad y el tratamiento concreto que recibe cada colectivo de pacientes. Además de descomponerlo en tres dimensiones, es interesante hacer la diferenciación entre calidad de vida objetiva y subjetiva.
La primera hace referencia a los componentes de cada una de las tres dimensiones que pueden ser cuantificables en función de criterios externos y la segunda en función de la valoración que cada persona hace de su propia experiencia física, emocional y social.

Es por ello, que al pensar en calidad de vida, debería de estar presente el concepto de desarrollo tecnológico en armonía con la calidad de vida que cada individuo experimenta en su rutina cotidiana. Del equilibrio entre ambos componentes, los individuos gozarían de un bienestar en su comunidad, desde el plano individual hacia el social.

El desfase que se puede presentar entre la ciencia, progreso tecnológico y la calidad de vida son temas que pueden integrar la definición de políticas públicas promovidas e impulsadas por cada gobierno, en beneficio de sus ciudadanos. Su evaluación, permanente y acorde con los lineamientos establecidos por las Organizaciones internacionales como la ONU y la Organización Mundial de la Salud (OMS), haría más clara la estrategia que se debe promover para asegurar la armonía entre ciencia, progreso tecnológico y la calidad de vida de las personas. Los eslabones comienzan a construirse desde el ámbito del gobierno municipal, estatal y federal.

\section{Pertinencia de los estudios EN CALIDAD DE VIDA}

En este sentido Colobrans (2001) nos dice que el primer paso hacia una valoración con éxito de la calidad de vida en la investigación, deberá consistir en clarificar la definición de ésta y en enumerar las dimensiones que la componen. La herramienta básica y 
estándar de medida es el cuestionario, bien sea autoadministrado o con apoyo de un entrevistador entrenado.

Dado que un cuestionario pretende medir variables intangibles no calculables por medios directos, éste debe cumplir tres requisitos que determinan su eficacia: validez, fiabilidad y sensibilidad a los cambios.

- Por validez de un cuestionario entendemos la capacidad de este para medir con la mayor exactitud posible la variable objeto de estudio.

- Además debe de haber un alto grado de fiabilidad o repetitividad en los resultados en circunstancias similares, es decir, ante la misma situación el cuestionario debe de dar los mismos valores de medida.

- Por último, un cuestionario debe ser sensible a los cambios o modificaciones en las variables que se están midiendo, lo que nos permite la comparabilidad de resultados entre distintos momentos; éste es un criterio estadístico importante, pues en los cambios ocurridos en una persona a lo largo del tiempo está el verdadero interés de la investigación de la calidad de vida, siendo el valor de partida individual de la primera medición el valor de referencia para los resultados de las sucesivas mediciones.

\section{Desarrollo humano $Y$ CALIDAD DE VIDA}

Las contribuciones del PNUD, realizadas bajo el liderazgo de Mahbub ul Haq y Amartya Sen, han llamado la atención de la comunidad internacional sobre la necesidad de construir consensos en torno a la idea básica de que la generación de rique- za no es un fin en sí mismo, sino un medio privilegiado para impulsar el proceso conducente a la ampliación de opciones y capacidades de las personas en todas las esferas. De acuerdo con esta perspectiva, la posesión de bienes materiales o la satisfacción de los deseos del individuo no constituyen los ámbitos adecuados para medir y evaluar el bienestar.

El espacio ideal para hacerlo es el de las capacidades para lograr aquello que los individuos valoran. En este marco, en diversos foros se ha propuesto que el objetivo principal de las políticas públicas es el de contribuir a mejorar la calidad de vida y el bienestar de la población mediante el diseño y la instrumentación de programas encaminados a ampliar las capacidades y opciones de las personas y aprovechar todo su potencial.

Desde esta perspectiva se reconoce que en todos los niveles de desarrollo hay algunas capacidades y opciones que son esenciales para que las personas participen en la sociedad, contribuyan a ella y se desarrollen plenamente. Algunas de estas capacidades básicas, sin las cuales muchas otras oportunidades permanecerían inaccesibles, son: la de permanecer vivo y gozar de una vida larga y saludable; la de adquirir conocimientos, comunicarse y participar en la vida de la comunidad; y la de contar con acceso a los recursos necesarios para disfrutar de un nivel de vida digno.

Algunas otras capacidades y opciones relevantes incluyen la libertad política, económica, social y cultural; la disponibilidad de oportunidades con fines productivos o de creación; el respeto por sí mismo; el ejercicio pleno de los Derechos Humanos; y la 
conciencia de pertenecer a una comunidad. De acuerdo con esta perspectiva, el desarrollo humano no consiste solamente en contar con todas esas capacidades y ampliarlas permanentemente, sino también en procurarlas de manera productiva, equitativa, sustentable y participativa.

Un desarrollo humano inequitativo y contrastante se refleja, entre otros aspectos, en comportamientos demográficos muy diferenciados. Diversos estudios revelan que factores demográficos tales como el crecimiento natural de la población, los niveles de fecundidad y la razón de dependencia demográfica guardan estrecha correspondencia con el grado de desarrollo humano alcanzado.

Ello sugiere que la evolución demográfica de países como México depende, en buena medida, de la ampliación de las libertades, capacidades y opciones de las personas y del control que tengan sobre las decisiones clave para configurar su destino. El desarrollo humano es una condición indispensable para garantizar que las personas ejerzan con plenitud sus derechos. A su vez, el disfrute de otras muchas libertades y derechos de ciudadanía social se ve favorecido por la expansión de las capacidades de las personas para cristalizar sus preferencias reproductivas.

El Programa de las Naciones Unidas para el Desarrollo (PNUD) publica año con año el Informe sobre Desarrollo Humano. En cada nuevo informe anual, el PNUD se ha preocupado por explorar y reflexionar sobre el devenir de diversos fenómenos relevantes que influyen en el bienestar y la calidad de vida de la población. Asimismo, ha introducido nuevos conceptos, promovido debates y formulado propuestas que apuntan hacia un nuevo paradigma de desarrollo, al tiempo que ha propiciado un mayor interés por la evaluación de los esfuerzos realizados en materia de desarrollo humano. De hecho, desde su lanzamiento, la propuesta conceptual y metodológica del llamado Índice de Desarrollo Humano (IDH) ha sido enriquecida en cada publicación anual y en el curso de la década fueron incorporados otros índices, como son el de Desarrollo relativo al Género (IDG), el Índice de Potenciación de Género (IPG) y el Índice de Pobreza Humana (IPH).

Como se sabe, es difícil contar con una medida que capture adecuada e integralmente la compleja realidad que el paradigma del desarrollo humano busca aprehender. No obstante, el IDH es una medida innovadora y útil que pone de manifiesto que el bienestar y el ingreso no son dimensiones equiparables. En esencia, se trata de un indicador compuesto, comparable internacionalmente, que combina:

- La longevidad: medida mediante la esperanza de vida al nacer;

- El logro educacional, a través de la alfabetización de adultos y la matrícula combinada de varios niveles educativos; $\mathrm{y}$

- El nivel de vida, mediante el PIB per cápita anual ajustado (paridad del poder adquisitivo en dólares).

$\mathrm{Al}$ incluir los logros en los tres campos indicados, el IDH ha logrado abrir el abanico de indicadores que se pueden utilizar en la medición del desarrollo, al tiempo que ha buscado medir el progreso socioeconómico de casi todos los países del mundo a través de unos cuantos indicadores suficientemen- 
te homogéneos y relativamente universales. En este sentido, se puede decir que la propia construcción del IDH prescribe el diseño e instrumentación de políticas públicas para combinar los esfuerzos económicos dirigidos a incrementar los ingresos de las personas y una distribución más equitativa de éstos con aquéllos orientados a abatir los rezagos en materia de educación y salud.

Para construir este indicador, es necesario establecer valores mínimos y máximos en cada dimensión crítica del desarrollo humano, los cuales, al ser normalizados, se transforman a una escala lineal que va de 0 a 1 y que indica la distancia socioeconómica que tiene que ser recorrida para alcanzar ciertas metas $\mathrm{u}$ objetivos deseables. Los indicadores de aquellas tres dimensiones se combinan en un índice global (el IDH) mediante un promedio aritmético de ellos mismos.

Cada país puede ser ubicado en algún punto de esa escala, de modo que la diferencia entre el valor máximo posible y el valor registrado indica el grado de insuficiencia en cada uno de estos indicadores. De esta manera, cuánto más cercano esté un país de un IDH con valor igual a 1 , tanto menor es la distancia que le queda por recorrer.

El IDH es un valioso instrumento de comparación entre distintos países y dentro de un mismo país. Sin embargo, conviene reconocer que el IDH no puede reflejar en toda su magnitud la complejidad del concepto de desarrollo humano. Por esta razón, la información proporcionada por el IDH y otros índices semejantes propuestos por el PNUD suelen complementarse mediante el análisis de otras muchas dimensiones relevantes del desarrollo humano. De cualquier forma, este tipo de aproximaciones presen- ta una visión poderosa de las condiciones básicas en que viven las personas e, incluso, pueden contribuir a orientar las políticas públicas hacia objetivos concretos, generar debate e informar a la opinión pública sobre las diversas cuestiones vinculadas con el desarrollo social. De hecho, una de las principales ventajas del índice de desarrollo humano es la posibilidad de establecer una jerarquía casi visual sobre quien está mejor y quien peor.

El desarrollo de un país no puede ser entendido solamente como crecimiento económico general. El desarrollo de un país se encuentra en cada uno de sus habitantes y en las posibilidades que ellos tienen para vivir una vida en la que puedan realizar a plenitud su potencial como seres humanos.

Así podemos concluir que el desarrollo humano consiste en la libertad de que gozan los individuos para elegir entre distintas opciones y formas de vida. Los factores que permiten a las personas ser libres en ese sentido son la posibilidad de alcanzar una vida larga y saludable, poder adquirir conocimientos individual y socialmente valiosos, y tener la oportunidad de obtener los recursos necesarios para disfrutar de un nivel de vida decoroso.

En el núcleo del concepto de desarrollo humano se encuentran las personas y sus oportunidades, no la riqueza que poseen, el ingreso que devengan o las mercancías y los servicios que consumen.

\section{Conclusiones}

La ciencia y el progreso tecnológico, responden a una necesidad de conocimiento e innovación, aunque esto pueda implicar un 
desconocimiento del impacto que tiene esa novedad en conocimiento en la calidad de vida de los individuos.

Uno de los problemas a los que se enfrenta el estudio de la Calidad de Vida es al hecho de no estar establecida una única definición consensuada y delimitada conceptualmente. Cuando se progresa y, en consecuencia, se produce un desarrollo tecnológico, no necesariamente trasciende en la calidad de vida de las personas. Hay ocasiones en que el riesgo de progresar en el saber tecnológico implica una afectación a la calidad de vida y bienestar de los individuos.

La manera de equilibrar los factores, puede ser desde la instrumentación de políticas públicas diseñadas y evaluadas por los distintos niveles de gobierno (municipal, estatal y federal). Esta evaluación, se puede realizar siguiendo los indicadores sugeridos por la comunidad internacional.

\section{REFERENCIAS}

Augsto B., C. (2006). Metodología de la Investigación. Para administración, economía, humanidades y ciencias sociales ( $2^{\underline{a}}$ ed.). México: Pearson Prentice Hall.

Bunge, M. (2000). La ciencia, su método y su filosofía. Siglo XXI Editores.

Colobrans, J. (2001). El doctorado organizado. La gestión del conocimiento aplicada a la investigación. España: Mira editores, España.

Consejo nacional de Ciencia y Tecnología. Recuperado el 22 de agosto de 2008 de: http://www.conacyt.mx/.

Consejo Estatal de Ciencia y Tecnología de Jalisco, Fondo Mixto. Recuperado el 22 de agosto de 2008 de: http://www.conacyt.mx/Fondos/FondosMixtos.html.

Eco, U. (2000). Cómo se hace una tesis. Técnicas y procedimientos de estudio, investigación y escritura. Barcelona, España.
Instituto de Investigaciones Jurídicas. Recuperado el 22 de agosto de 2008 de: http://info4.juridicas.unam.mx/ijure/ fed $/ 9 /$.

Munch, L. \& Ángeles, E. (2005). Métodos y técnicas de investigación ( $2^{\underline{a}}$ ed.). México: Trillas.

Méndez, C. (2005). Metodología. Diseño y desarrollo del proceso de investigación (3a ed.). Colombia: McGrawHill.

Secretaría de Gobernación Plan Nacional de Desarrollo. Recuperado el 22 de agosto de 2008 de: http://pnd.presidencia.gob. $\mathrm{mx} /$.

Zapata, O. (2005). La aventura del pensamiento crítico. Herramientas para elaborar tesis $e$ investigaciones socioeducativas. México: Editorial Pax. 